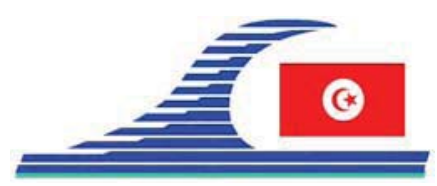

\author{
Conférence Méditerranéenne Côtière et Maritime \\ EDITION 1, HAMMAMET, TUNISIE (2009) \\ Coastal and Maritime Mediterranean Conference \\ Disponible en ligne - http://www.paralia.fr-Available online
}

\title{
Nouvelle méthode de monitoring in situ du phytoplancton : analyse automatisée à haute fréquence et à l'échelle individuelle des cellules
}

\author{
Melilotus THYSSEN ${ }^{\mathbf{1}}$, Gérald GREGORI ${ }^{1}$, \\ Anthony MALKASSIAN ${ }^{1}$, Michel DENIS ${ }^{1}$
}

1. Université de la Méditerranée, Centre d'Océanologie de Marseille, Laboratoire de Microbiologie, Géochimie et Écologie Marines, CNRS UMR6117, 163 avenue de Luminy, Case 901, 13288 Marseille cedex 09, France.

melilotus.thyssen@univmed.fr ; gerald.gregori@univmed.fr ;

anthony.malkassian@etumel.univmed.fr ;michel.denis@univmed.fr

\section{Résumé :}

Une nouvelle méthode d'étude in situ du phytoplancton (THYSSEN et al., 2008a) a été élaborée pour étudier la variabilité à court terme et l'hétérogénéité spatiale de la distribution du phytoplancton marin. Cette approche émergente utilise un cytomètre en flux submersible et automatisé (Cytosub, www.cytobuoy.com). La variabilité à court terme de l'assemblage phytoplanctonique a été étudiée dans la baie de Marseille (Méditerranée nord occidentale) où l'instrument, immergé à 2 m les étés 2005 et 2006, était déclenché toutes les $30 \mathrm{~min}$. Les 7 groupes de cellules résolus dans la classe de taille 1-50 $\mu \mathrm{m}$ se comportaient comme des entités indépendantes et pourraient donc être assimilés à des groupes fonctionnels de réponse.

L'hétérogénéité spatiale de la distribution du phytoplancton de surface a été étudiée le long de la radiale Açores-Bretagne en avril 2007. Le Cytosub, placé à bord d'une goélette de $33 \mathrm{~m}$ (Fetia Ura, www.seanergies.com), analysait l'eau de mer pompée sous le bateau chaque $15 \mathrm{~min}$ (résolution spatiale : 2,8 km). Des relations furent établies entre la distribution des 6 groupes de cellules identifiés et les différentes masses d'eau échantillonnées. La cytométrie en flux in situ et automatisée est un outil puissant pour étudier à haute fréquence les assemblages phytoplanctoniques. Les développements en cours visent à étendre les capacités de cette approche à l'ensemble des microorganismes marins et à automatiser le traitement des enregistrements.

\section{Mots-clés :}

Phytoplancton - Cytométrie en flux in situ - Automatisation - Haute fréquence 


\section{Introduction}

$\mathrm{Du}$ fait de la grande complexité des assemblages liée à une très importante diversité phylogénétique et à une forte variabilité de taille, de concentration et de physiologie, le phytoplancton n'est généralement pas correctement échantillonné, essentiellement du fait du manque de techniques appropriées (SMAYDA, 1998 ; BARETTA et al., 1998). Pour nous affranchir des limitations des méthodes conventionnelles, nous avons développé une nouvelle approche (THYSSEN et al., 2008a) mettant en œuvre un nouvel instrument (Cytosub, www.cytobuoy.com) spécialement conçu pour l'étude in situ du phytoplancton, à l'échelle individuelle des cellules et de façon automatisée. Cet instrument a aussi la particularité d'enregistrer les profils des signaux (diffusion, fluorescence) générés par les cellules lors de leur interception par le faisceau laser. Nous avons étudié la variabilité à court terme de la distribution du phytoplancton en déployant le Cytosub dans la baie de Marseille au cours des étés 2005 et 2006 (THYSSEN et al., 2008b). L'étude de l'hétérogénéité spatiale de la distribution du phytoplancton de surface a fait l'objet d'une campagne à bord d'un voilier reliant les Açores à la Bretagne en avril 2007 (THYSSEN et al., 2009). Les résultats présentés sont issus du travail de thèse de THYSSEN (2008) et ont fait l'objet de plusieurs publications (THYSSEN et al., 2008 a,b, 2009).

\section{Résultats et discussion}

\subsection{Variabilité à court terme}

Le Cytosub était déployé dans la baie de Marseille (Méditerranée nord occidentale) à 2 $\mathrm{m}$ de profondeur à l'intérieur d'une base nautique. Un câble étanche assurait son alimentation en énergie et le transfert des données en temps quasi-réel à l'ordinateur pilote.

Les analyses par le Cytosub étaient déclenchées toutes les 30 min suivant 2 protocoles successifs favorisant respectivement l'analyse des plus petites cellules $(>1 \mu \mathrm{m})$ et des plus grandes avec dans ce cas un seuil empêchant la saturation des mémoires tampons par les signaux des plus petites cellules. Sept groupes de cellules, désignés de $\mathrm{C} 1$ à $\mathrm{C} 7$, ont été résolus sur la base de leurs propriétés optiques et des profils des signaux. Les cycles cellulaires ont été déterminés en combinant les variations haute fréquence de fluorescence, de taille et d'abondance (DURAND \& OLSON, 1996; VAULOT \& MARIE, 1999).

Un résultat majeur de cette étude est que les groupes de cellules se comportaient comme des entités indépendantes à la suite de changements du rapport N/P ou de coups de vent. A ce titre, ils peuvent être considérés comme des groupes fonctionnels de réponse (HOPPER et al., 2002). Des études supplémentaires sont nécessaires pour déterminer la réponse de ces groupes à d'autres changements environnementaux et ouvrir la voie à l'identification de bio-indicateurs de changements environnementaux spécifiques. 
La combinaison de l'étude in situ à haute fréquence de ces groupes fonctionnels avec l'identification d'espèces constituera un outil particulièrement puissant pour une meilleure compréhension du fonctionnement des assemblages phytoplanctoniques.

\subsection{Hétérogénéité spatiale}

Pour documenter l'hétérogénéité spatiale de la distribution du phytoplancton avec une résolution compatible avec la sub-méso-échelle (de 1 à $10 \mathrm{~km}$ ), le Cytosub a été installé à l'intérieur d'une goélette de $33 \mathrm{~m}$ (Fetia Ura, http://www.seanergiesoceanes.abcsalles.com/prive/fr/fiche.php? $\mathrm{n}=10617$ ) pour la campagne ACYPHAR (Atlantic flow CYtometry spatial study of PHytoplankton through Automated Recording, 14 - 23 avril 2007) entre Horta $\left(38.6^{\circ} \mathrm{N}-28.6^{\circ} \mathrm{W}\right.$, île de Faïal, Açores) et Lorient $\left(47.6^{\circ} \mathrm{N}-3.6^{\circ} \mathrm{W}\right.$, Bretagne), (voir THYSSEN et al., 2009 pour plus de détails sur le dispositif expérimental).

Six groupes de cellules furent résolus par cytométrie en flux et 4 masses d'eau distinctes furent identifiées le long du parcours du voilier sur la base des relations températuresalinité déduites des données collectées simultanément par un capteur autonome de température, salinité et pression (CTD, SBE 37SMP). Les relations d'abondance étaient complexes et illustraient une forte hétérogénéité spatiale comme attendu. Une variabilité à court terme était superposée à des variations de tendance à plus grande échelle. Ces deux modes de variation ont été isolés par des traitements mathématiques appropriés. Des calculs d'auto-corrélation ont été appliqués au signal de variabilité à court terme pour mettre en évidence les périodicités liées au cycle cellulaire. L'analyse à haute fréquence a été aussi indispensable pour obtenir une résolution spatiale meilleure que 3 $\mathrm{km}$ et documenter la distribution du phytoplancton de surface à la sub-méso-échelle.

\section{Conclusion}

L'analyse à haute fréquence des assemblages phytoplanctoniques à l'aide de la cytométrie en flux submersible et automatisée ouvre la voie à une meilleure compréhension du fonctionnement de l'écosystème océanique. Elle est particulièrement adaptée à la réalisation de séries temporelles, à l'étude d'impacts liés aux aménagements côtiers. Elle a également sa place en aquaculture et dans les sytèmes d'observation de l'océan global qu'elle pourrait compléter enfin avec de réelles informations biologiques en étant déployée soit à des points fixes soit à bord de navires d'opportunité. Les données ainsi collectées seront particulièrement précieuses pour calibrer les algorithmes développés pour la télédétection des signaux de chlorophylle. L'assimilation des groupes cytométriques de cellules à des groupes fonctionnels de réponse doit faire l'objet de recherches complémentaires. 
Meilleures pratiques environnementales en ingénierie côtière et maritime

\section{Références}

BARETTA J.W., BARETTA-BEKKER J.G., RUARDIJ P. (1998). Data needs for ecosystem modelling. I.C.E.S. J. Mar. Sci. 55, pp 756-766.

DURAND M.D., OLSON R.J. (1996). Contributions of phytoplankton light scattering and cell concentration changes to diel variations in beam attenuation in the Equatorial Pacific from flow cytometric measurements of pico-, ultra- and nanoplankton. Deep Sea Res. (II) 43, pp 891-906.

SMAYDA T.J. (1978). From phytoplankton to biomass. In: Sournia, A. (ed.), Phytoplankton Manual. Monographs on Oceanographic Methodology 6. UNESCO, Paris, pp 273-279.

THYSSEN M. (2008). Analyse à haute fréquence spatiale et temporelle du phytoplancton à l'aide de la cytométrie en flux automatisée et immergeable. Thèse, Université de la Méditerranée, $222 \mathrm{p}$.

THYSSEN M., TARRAN G.A., ZUBKOV M.V., HOLLAND R.J., GRÉGORI G., BURKILL P.H., DENIS M. (2008a). The emergence of automated high frequency flow cytometry: revealing temporal and spatial phytoplankton variability. J. Plank. Res. 30, pp 333-343.

THYSSEN M., MATHIEU D., GARCIA N., DENIS M. (2008b). Short-term variation of phytoplankton assemblages in Mediterranean coastal waters recorded with an automated submerged flow cytometer. J. Plank. Res. 30, pp 1027-1040.

THYSSEN M., GARCIA N., DENIS M. (2009). Sub meso scale phytoplankton distribution in the North East Atlantic surface waters determined with an automated flow cytometer. Biogeosciences 6, pp 569-583.

VAULOT D., MARIE D. (1999). Diel variability of photosynthetic picoplankton in the equatorial Pacific. J. Geophys. Res. 104, pp 3297-3310. 